\title{
Kirchhoff板弯曲问题的改进的复变量无单元 Galerkin方法
}

\author{
王斌骅 ${ }^{1}$, 马永其 ${ }^{1,2}$, 冯伟 ${ }^{1}$, 程玉民 ${ }^{1 *}$ \\ 1. 上海大学上海市应用数学和力学研究所, 上海 200072 ; \\ 2. 上海大学力学系, 上海 200444 \\ *联系人, E-mail: ymcheng@shu.edu.cn \\ 收稿日期: 2016-10-19; 接受日期: 2016-12-13; 网络出版日期: 2017-07-14 \\ 国家自然科学基金(编号: 11571223, U1433104)资助项目
}

\begin{abstract}
摘要基于改进的复变量移动最小二乘法, 建立了Kirchhoff板弯曲问题的改进的复变量无单元Galerkin方法. 相对于移动最小二乘法, 改进的复变量移动最小二乘法采用一维基函数建立二维问题的逼近函数, 提高了形 函数计算效率. 由改进的复变量移动最小二乘法建立Kirchhoff板的挠度逼近函数, 根据Kirchhoff板弯曲问题的 Galerkin弱形式建立离散方程, 并应用罚函数法施加本质边界条件, 推导了Kirchhoff板弯曲问题的改进的复变量 无单元Galerkin法的公式. 通过对 4 个典型算例进行计算和分析, 说明了本文建立的Kirchhoff板弯曲问题的改进 的复变量无单元Galerkin方法的有效性, 并通过分析数值解的精度对本文方法中如何选取合适的基函数、权函 数、影响域比例参数、节点分布和罚因子进行了讨论. 数值算例说明了本文方法具有较好的收玫性和较高的 计算精度。
\end{abstract}

\begin{abstract}
关键词无网格方法, 改进的复变量移动最小二乘法, 复变量无单元Galerkin方法, 改进的复变量无单元 Galerkin方法, Kirchhoff板
\end{abstract}

PACS: 45.10.-b, 46.15.-x, 46.25.-y, 46.70.De

\section{1 引言}

无网格方法作为一种数值方法, 是继有限元法之 后科学和工程计算方法的重要发展. 无网格方法在处 理问题时只需要节点信息, 不需要对求解域进行网格 划分, 而且在计算过程中可以根据需要在某一区域增 加或减少节点, 便于进行自适应计算, 同时也能提高
局部区域的计算精度. 板弯曲问题是力学和工程领域 重要的研究课题之一, 但目前对板弯曲问题无网格方 法的研究还比较少, 进一步研究和发展板弯曲问题的 无网格方法是必要的.

关于板弯曲问题的无网格方法, Hein ${ }^{[1]}$ 将扩散单 元法用于Kirchhoff板的分析; Krysl和Belytschko ${ }^{[2]}$ 应 用无单元Galerkin方法(Element-Free Galerkin Method,

引用格式: 王斌骅, 马永其, 冯伟, 等. Kirchhoff板弯曲问题的改进的复变量无单元Galerkin方法. 中国科学: 物理学力学 天文学, 2017, 47: 094601 Wang B H, Ma Y Q, Feng W, et al. The improved complex variable element-free Galerkin method for the analysis of Kirchhoff plates (in Chinese). Sci Sin-Phys Mech Astron, 2017, 47: 094601, doi: 10.1360/SSPMA2016-00437 
$\mathrm{EFG})$ 求解了薄板问题; $\mathrm{Li}^{\text {等人 }}{ }^{[3]}$ 用重构核粒子法分析 了薄壳的大变形问题; Liu等人 ${ }^{[4,5]}$ 用无网格方法研究 了薄板的静态和自由震动问题; Sladek等人 ${ }^{[6,7]}$ 用无网 格方法对板的大变形问题进行了数值模拟; Chen等 人 ${ }^{[8]}$ 建立了复变量重构核粒子法, 以此方法建立形函 数, 提出了Kirchhoff板弯曲问题的复变量重构核粒子 法 ${ }^{[9]}$.

移动最小二乘法(Moving Least-Squares Approximation, MLS)被广泛应用于无网格方法的形函数构造, 其泛函为加权最佳平方逼近形式, 因而有较高的计算 精度; 程玉民等人 ${ }^{[10]}$ 提出了改进的移动最小二乘法, 通 过对基函数的正交化, 克服了移动最小二乘法易形成 病态方程组的缺点. 为了解决无单元 Galerkin方法等 无网格方法不能直接施加本质边界条件的问题, Wang 等人 ${ }^{[11]}$ 和Sun等人 ${ }^{[12]}$ 建立了移动最小二乘插值法, 并 研究了其误差分析理论. 为了解决无单元Galerkin方 法等无网格方法计算量大的问题, Cheng等人 ${ }^{[13-15]}$ 将 复变量理论引入移动最小二乘法, 提出了复变量移 动最小二乘法(Complex Variable Moving Least-Squares Approximation, CVMLS), 其优点是减少了试函数中所 含待定系数的个数, 所形成的二维问题的无网格方法 可取较少的节点. 这样, 对任一场点来说, 影响域中所 含的最小节点数减少了, 进而在整个求解域中所选取 的节点数也就可以大大减少. 与基于移动最小二乘法 的无网格方法相比, 在节点分布相同时, 基于复变量移 动最小二乘法的无网格方法可提高计算精度; 在计算 精度相近时, 基于复变量移动最小二乘法的无网格方 法具有更高的计算速度. 针对复变量移动最小二乘法 中泛函的数学和物理意义不明确的问题, Ren等人 ${ }^{[16]}$ 建立了具有明确数学和物理意义的泛函, 提出了改进 的复变量移动最小二乘法(Improved Complex Variable Moving Least-Squares Approximation, ICVMLS).

基于移动最小二乘法形成的无单元Galerkin方法 是目前研究和应用最广泛的无网格方法之一. 将改进 的移动最小二乘法和Galerkin弱形式结合, Zhang等人 提出了瞬态热传导 ${ }^{[17]}$ 、波动方程 ${ }^{[18]}$ 和弹性动力学 ${ }^{[19]}$ 的改进的无单元Galerkin方法; Peng等人 ${ }^{[20]}$ 应用改进的 无单元Galerkin方法, 分析了三维黏弹性问题. Cheng 等人 ${ }^{[21]}$ 应用移动最小二乘插值法建立形函数, 提出了 改进的无单元Galerkin方法, 分析了非线性大变形问 题. Sun等人 ${ }^{[22]}$ 提出了基于非奇异权函数的改进的无
单元Galerkin方法, 分析了非线性弹塑性问题. 改进 的无单元Galerkin方法具有配点少、精度高、计算速 度快和不易形成病态方程组的优点. Peng等人基于 复变量移动最小二乘法, 结合 Galerkin弱形式建立了 弹性力学 ${ }^{[23]}$ 和弹塑性力学 ${ }^{[24]}$ 的复变量无单元Galerkin 方法(Complex Variable Element-Free Galerkin Method, CVEFG). Cheng等人建立了弹性动力学 ${ }^{[25]}$ 、黏弹性力 学 ${ }^{[26]}$ 、反问题 ${ }^{[27]}$ 和弹塑性大变形问题 ${ }^{[28]}$ 的复变量无 单元Galerkin方法. 在节点分布相同时, 复变量无单元 Galerkin方法比传统的无单元Galerkin方法具有更高的 计算精度; 在计算精度相近时, 复变量无单元Galerkin 方法则比传统的无单元Galerkin方法布置节点少, 从 而提高了计算效率. Deng等人 ${ }^{[29]}$ 应用复变量移动最 小二乘插值法(Interpolating Complex Variable Moving Least-Squares Approximation), 提出了温度场问题的插 值型复变量无单元Galerkin方法(Interpolating Complex Variable Element-Free Galerkin Method). Cheng等人 ${ }^{[30]}$ 应用复变量移动最小二乘插值法, 提出了二维弹塑 性问题的插值型复变量无单元Galerkin方法. Ren等 人 $^{[31]}$ 采用改进的复变量移动最小二乘法建立形函数, 提出了弹性力学的新的改进的复变量无单元Galerkin 方法(Improved Complex Variable Element-Free Galerkin Method, ICVEFG).

本文基于改进的复变量移动最小二乘法建立 Kirchhoff板的挠度逼近函数, 根据Kirchhoff板弯曲问 题的Galerkin弱形式建立离散方程, 并应用罚函数法 来施加本质边界条件, 提出了Kirchhoff板弯曲问题的 改进的复变量无单元Galerkin方法. 数值算例说明了 本文建立的Kirchhoff板弯曲问题的改进的复变量无 单元Galerkin方法的有效性, 并讨论了基函数、权函 数、影响域比例参数、节点分布和罚因子对本文方 法数值解精度的影响.

\section{Kirchhoff板的基本方程}

图 1所示为一厚度为 $h$ 的等厚度Kirchhoff板, 板的 中面为 $O x_{1} x_{2}$ 面, $x_{3}$ 轴垂直于中面, 作用在板上的横向 单位载荷为 $q\left(x_{1}, x_{2}\right)$. 在 $O x_{1} x_{2}$ 面上, 板的区域为 $\Omega$, 其边 界为 $\Gamma$.

根据Kirchhoff板的理论, 平行于未变形中面的位 移 $u$ 和 $v$ 的表达式为 


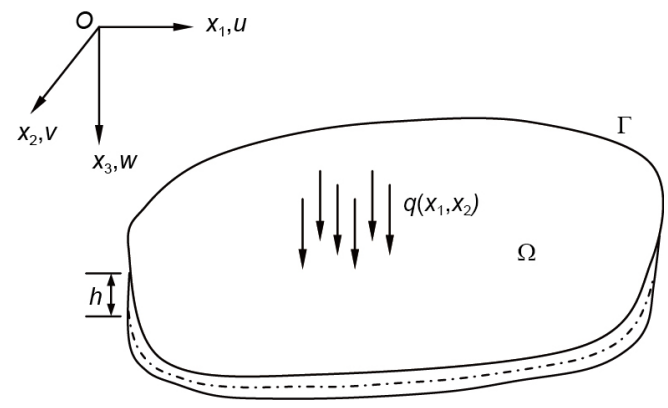

图 1 受横向分布载荷的Kirchhoff板

Figure 1 Kirchhoff plate subjected to transverse distributed load.

$u=-x_{3} \frac{\partial w}{\partial x_{1}}$

$v=-x_{3} \frac{\partial w}{\partial x_{2}}$

其中 $w$ 表示Kirchhoff板中面在 $x_{3}$ 方向的位移, 是Kirchhoff板弯曲的场点挠度.

各向同性和各向异性的Kirchhoff板的挠度控制方 程为

$D_{0} \nabla^{4} w\left(x_{1}, x_{2}\right)=q\left(x_{1}, x_{2}\right),\left(x_{1}, x_{2}\right) \in \Omega$,

其中 $\nabla^{4}(\cdot)$ 是双调和算子,

$\nabla^{4}(\cdot)=\frac{\partial^{4}(\cdot)}{\partial x_{1}^{4}}+2 \frac{\partial^{4}(\cdot)}{\partial x_{1}^{2} \partial x_{2}^{2}}+\frac{\partial^{4}(\cdot)}{\partial x_{2}^{4}}$,

$D_{0}$ 是Kirchhoff 板的弯曲刚度，

$D_{0}=\frac{E h^{3}}{12\left(1-v^{2}\right)}$,

$E$ 是杨氏模量, $v$ 是泊松比.

本质边界条件为

$w=\bar{w},\left(x_{1}, x_{2}\right) \in \Gamma_{u_{1}}$,

$\frac{\partial w}{\partial \boldsymbol{n}}=\bar{\theta}_{n},\left(x_{1}, x_{2}\right) \in \Gamma_{u_{2}}$,

自然边界条件为

$M_{n}=\bar{M}_{n},\left(x_{1}, x_{2}\right) \in \Gamma_{t_{1}}$,

$V_{n}=\bar{V}_{n},\left(x_{1}, x_{2}\right) \in \Gamma_{t_{2}}$,

其中 $M_{n}$ 是弯矩, $V_{n}$ 是实际剪力; $\bar{w}$ 是本质边界 $\Gamma_{u_{1}}$ 上的挠 度, $\bar{\theta}_{n}$ 是本质边界 $\Gamma_{u_{2}}$ 上切向的转角, $\bar{M}_{n}$ 是自然边界 $\Gamma_{t_{1}}$ 上
的弯矩, $\bar{V}_{n}$ 是自然边界 $\Gamma_{t_{2}}$ 上的法向等效剪力; $\boldsymbol{n}$ 是自然 边界 $\Gamma$ 的外法线方向向量.

\section{3 改进的复变量移动最小二乘法}

改进的复变量移动最小二乘法的逼近函数取为

$$
\begin{aligned}
w^{h}(z) & =w_{1}^{h}(z)+\mathrm{i} w_{2}^{h}(z) \\
& =\sum_{i=1}^{m} p_{i}(z) a_{i}(z)=\boldsymbol{p}^{\mathrm{T}}(z) \boldsymbol{a}(z), \\
z=x_{1} & +\mathrm{i} x_{2} \in \Omega,
\end{aligned}
$$

其中 $w^{h}(z)$ 是函数 $w(z)$ 的逼近函数, 其中, $\boldsymbol{p}^{\mathrm{T}}(z)=\left(p_{1}(z)\right.$, $\left.p_{2}(z), \cdots, p_{m}(z)\right)$ 是基向量, $\boldsymbol{a}^{\mathrm{T}}(z)=\left(a_{1}(z), a_{2}(z), \cdots\right.$, $\left.a_{m}(z)\right)$ 是相应的系数向量, $m$ 是基函数的个数.

本文采用的基函数为

二次多项式基函数 $(m=3)$,

$\boldsymbol{p}^{\mathrm{T}}=\left(1, z, z^{2}\right)$,

三次多项式基函数 $(m=4)$,

$\boldsymbol{p}^{\mathrm{T}}=\left(1, z, z^{2}, z^{3}\right)$,

四次多项式基函数 $(m=5)$,

$\boldsymbol{p}^{\mathrm{T}}=\left(1, z, z^{2}, z^{3}, z^{4}\right)$,

在点 $z$ 影响域内的局部逼近定义为

$w^{h}(z, \widehat{z})=\sum_{i=1}^{m} \bar{p}_{i}(z) \cdot a_{i}(z, \widehat{z})=\boldsymbol{p}^{\mathrm{T}}(z) \cdot \boldsymbol{a}(z, \widehat{z})$,

其中 $\widehat{z}$ 是影响域覆盖点 $z$ 的节点.

定义泛函

$$
\begin{aligned}
J= & \sum_{I=1}^{n} w_{h}\left(z-z_{I}\right)\left|w^{h}\left(z_{I}, z\right)-w\left(z_{I}\right)\right|^{2} \\
= & \sum_{I=1}^{n} w_{h}\left(z-z_{I}\right)\left(\sum_{i=1}^{m} p_{i}\left(z_{I}\right) \cdot a_{i}(z, \widehat{z})-w\left(z_{I}\right)\right) \\
& \times \overline{\left(\sum_{i=1}^{m} p_{i}\left(z_{I}\right) \cdot a_{i}(z, \widehat{z})-w\left(z_{I}\right)\right)},
\end{aligned}
$$

其中 $w_{h}\left(z-z_{I}\right)$ 是具有紧支性的权函数, $z_{I}(I=1,2, \ldots, n)$ 是影响域覆盖点 $z$ 的节点.

定义内积

$(f, g)_{z}=\sum_{I=1}^{n} w\left(z, z_{I}\right) f\left(z_{I}\right) \bar{g}\left(z_{I}\right), \forall f, g \in C^{0}(\bar{\Omega})$,

其中 $\bar{g}\left(z_{I}\right)$ 是 $g\left(z_{I}\right)$ 的共轭, $\bar{\Omega}=\Omega \cup \partial \Omega$. 函数 $f$ 的范 数为 


$$
\begin{aligned}
\|f\|_{z}= & {\left[(f, f)_{z}\right]^{\frac{1}{2}} . } \\
& \text { 式 }(15) \text { 可改写为 } \\
J= & (\boldsymbol{P a}(z, \widehat{z})-\boldsymbol{w}(\widehat{z}))^{\mathrm{T}} \boldsymbol{W}(z, \widehat{z}) \overline{(\boldsymbol{P a}(z, \widehat{z})-\boldsymbol{w}(\widehat{z}))} \\
= & \left\|\boldsymbol{p}^{\mathrm{T}}(z) \mathrm{a}(z, \widehat{z})-w\right\|_{z}^{2},
\end{aligned}
$$

其中

$\boldsymbol{w}(\widehat{z})=\left(w\left(z_{1}\right), w\left(z_{2}\right), \cdots, w\left(z_{n}\right)\right)^{\mathrm{T}}=\boldsymbol{Q} \boldsymbol{w}$,

$\boldsymbol{w}=\left(w_{1}\left(z_{1}\right), w_{2}\left(z_{1}\right), w_{1}\left(z_{2}\right), w_{2}\left(z_{2}\right), \cdots\right.$, $\left.w_{1}\left(z_{n}\right), w_{2}\left(z_{n}\right)\right)^{\mathrm{T}}$,

$\boldsymbol{Q}=\left[\begin{array}{ccccccccc}1 & \mathrm{i} & 0 & 0 & 0 & 0 & \cdots & 0 & 0 \\ 0 & 0 & 1 & \mathrm{i} & 0 & 0 & \cdots & 0 & 0 \\ 0 & 0 & 0 & 0 & 1 & \mathrm{i} & \cdots & 0 & 0 \\ \vdots & \vdots & \vdots & \vdots & \vdots & \vdots & \ddots & \vdots & \vdots \\ 0 & 0 & 0 & 0 & 0 & 0 & \cdots & 1 & \mathrm{i}\end{array}\right]_{n \times 2 n}$,

$\boldsymbol{P}=\left[\begin{array}{cccc}p_{1}\left(z_{1}\right) & p_{2}\left(z_{1}\right) & \cdots & p_{m}\left(z_{1}\right) \\ p_{1}\left(z_{2}\right) & p_{2}\left(z_{2}\right) & \cdots & p_{m}\left(z_{2}\right) \\ \vdots & \vdots & \ddots & \vdots \\ p_{1}\left(z_{n}\right) & p_{2}\left(z_{n}\right) & \cdots & p_{m}\left(z_{n}\right)\end{array}\right]_{n \times m}$,

$\overline{\boldsymbol{P}}=\left[\begin{array}{cccc}\bar{p}_{1}\left(z_{1}\right) & \bar{p}_{2}\left(z_{1}\right) & \cdots & \bar{p}_{m}\left(z_{1}\right) \\ \bar{p}_{1}\left(z_{2}\right) & \bar{p}_{2}\left(z_{2}\right) & \cdots & \bar{p}_{m}\left(z_{2}\right) \\ \vdots & \vdots & \ddots & \vdots \\ \bar{p}_{1}\left(z_{n}\right) & \bar{p}_{2}\left(z_{n}\right) & \cdots & \bar{p}_{m}\left(z_{n}\right)\end{array}\right]_{n \times m}$,

$$
\begin{aligned}
\boldsymbol{a}^{\mathrm{T}}(z, \widehat{z}) & =\left(a_{1}(z, \widehat{z}), a_{2}(z, \widehat{z}), \cdots, a_{m}(z, \widehat{z})\right) \\
& =\left(a_{1}, a_{2}, \cdots, a_{m}\right),
\end{aligned}
$$

$\boldsymbol{W}(z, \widehat{z})=$

$\left[\begin{array}{cccc}W\left(z-z_{1}\right) & 0 & \cdots & 0 \\ 0 & W\left(z-z_{2}\right) & \cdots & 0 \\ \vdots & \vdots & \ddots & \vdots \\ 0 & 0 & \cdots & W\left(z-z_{n}\right)\end{array}\right]_{n \times n}$

式(18)可写为

$J=\left\|a_{1} p_{1}+a_{2} p_{2}+\cdots+a_{m} p_{m}-w\right\|_{z}^{2}$,

其中 $\bar{p}_{i}=\bar{p}_{i}(z), i=1,2, \cdots, m$.

令 $w^{(1)}$ 为 $w$ 在空间 $\operatorname{span}\left(\bar{p}_{1}, \bar{p}_{2}, \cdots, \bar{p}_{m}\right)$ 的投影, 则

$J^{(1)}=\left\|w^{(1)}-w\right\|_{z}^{2}$

是泛函 $J$ 的最小值.
函数 $w$ 可写为

$w=w^{(1)}+w^{(2)}$,

其中 $w^{(2)} \perp \operatorname{span}\left(p_{1}, p_{2}, \cdots, p_{m}\right)$.

显然有

$\left(p_{i}, w^{(2)}\right)_{z}=0, i=1,2, \cdots, m$,

则

$$
\begin{aligned}
\left(p_{i}, w\right)_{z} & =\left(p_{i}, w^{(1)}\right)_{z}+\left(p_{i}, w^{(2)}\right)_{z} \\
& =\left(p_{i}, w^{(1)}\right)_{z}, \quad i=1,2, \cdots, m,
\end{aligned}
$$

于是

$$
\begin{aligned}
\left(p_{i}, w\right)_{z} & =\left(p_{i}, w^{(1)}\right)_{z} \\
& =\left(p_{i}, a_{1} p_{1}+a_{2} p_{2}+\cdots+a_{m} p_{m}\right)_{z},
\end{aligned}
$$

将式(31)写成矩阵形式为

$$
\begin{aligned}
& {\left[\begin{array}{cccc}
\left(p_{1}, p_{1}\right)_{z} & \left(p_{1}, p_{2}\right)_{z} & \cdots & \left(p_{1}, \bar{p}_{m}\right)_{z} \\
\left(p_{2}, p_{1}\right)_{z} & \left(p_{2}, p_{2}\right)_{z} & \cdots & \left(p_{2}, \bar{p}_{m}\right)_{z} \\
\vdots & \vdots & \ddots & \vdots \\
\left(p_{m}, p_{1}\right)_{z} & \left(p_{m}, p_{2}\right)_{z} & \cdots & \left(p_{m}, \bar{p}_{m}\right)_{z}
\end{array}\right]\left[\begin{array}{c}
\bar{a}_{1}(z, \widehat{z}) \\
\bar{a}_{2}(z, \widehat{z}) \\
\vdots \\
\bar{a}_{m}(z, \widehat{z})
\end{array}\right]} \\
& =\left[\begin{array}{c}
\left(p_{1}, w\right)_{z} \\
\left(p_{2}, w\right)_{z} \\
\vdots \\
\left(p_{m}, w\right)_{z}
\end{array}\right],
\end{aligned}
$$

即

$$
\left(\boldsymbol{P}^{\mathrm{T}} \boldsymbol{W}(z, \widehat{z}) \overline{\boldsymbol{P}}\right) \overline{\boldsymbol{a}}(z, \widehat{z})=\boldsymbol{P}^{\mathrm{T}} \boldsymbol{W}(z, \widehat{z}) \overline{\boldsymbol{w}}(\widehat{z}),
$$

式(33)的共轭为

$$
\left(\overline{\boldsymbol{P}}^{\mathrm{T}} \boldsymbol{W}(z, \widehat{z}) \boldsymbol{P}\right) \boldsymbol{a}(z, \widehat{z})=\overline{\boldsymbol{P}}^{\mathrm{T}} \boldsymbol{W}(z, \widehat{z}) \boldsymbol{w}(\widehat{z}),
$$

于是可得

$\boldsymbol{a}(z, \widehat{z})=\boldsymbol{A}^{-1}(z, \widehat{z}) \boldsymbol{B}(z, \widehat{z}) \boldsymbol{w}(\widehat{z})$,

其中

$$
\begin{aligned}
& \boldsymbol{A}(z, \widehat{z})=\overline{\boldsymbol{P}}^{\mathrm{T}} \boldsymbol{W}(z, \widehat{z}) \boldsymbol{P}, \\
& \boldsymbol{B}(z, \widehat{z})=\overline{\boldsymbol{P}}^{\mathrm{T}} \boldsymbol{W}(z, \widehat{z}) .
\end{aligned}
$$

那么, 由式(14)可得局部近似函数 $w^{h}(z, \widehat{z})$ 为

$$
w^{h}(z, \widehat{z})=\boldsymbol{\Phi}(z) \boldsymbol{w}(\widehat{z})=\sum_{I=1}^{n} \Phi_{I}(z) w\left(z_{I}\right),
$$

其中形函数 


$$
\begin{aligned}
\boldsymbol{\Phi}(z) & =\left(\Phi_{1}(z), \Phi_{2}(z), \cdots, \Phi_{n}(z)\right) \\
& =p^{\mathrm{T}}(z) \boldsymbol{A}^{-1}(z, \widehat{z}) \boldsymbol{B}(z, \widehat{z}) .
\end{aligned}
$$

由式(38)可得

$w_{1}^{h}(z)=\operatorname{Re}[\boldsymbol{\Phi}(z) \boldsymbol{w}(\widehat{z})]=\operatorname{Re}\left[\sum_{I=1}^{n} \Phi_{I}(z) w\left(z_{I}\right)\right]$,

$w_{2}^{h}(z)=\operatorname{Im}[\boldsymbol{\Phi}(z) w(\widehat{z})]=\operatorname{Im} \sum_{I=1}^{n}\left[\Phi_{I}(z) w\left(z_{I}\right)\right]$.

\section{Kirchhoff板弯曲问题的改进的复变量无 单元Galerkin方法}

定义广义应变向量

$$
\boldsymbol{\varepsilon}_{p}=\left[\begin{array}{c}
-\frac{\partial^{2} w}{\partial x_{1}^{2}} \\
-\frac{\partial^{2} w}{\partial x_{2}^{2}} \\
-2 \frac{\partial^{2} w}{\partial x_{1} \partial x_{2}}
\end{array}\right]=\boldsymbol{L} w
$$

和广义内力向量

$$
\boldsymbol{\sigma}_{p}=\left[\begin{array}{l}
M_{11} \\
M_{22} \\
M_{12}
\end{array}\right]=\boldsymbol{D} \boldsymbol{\varepsilon}_{p},
$$

其中 $L$ 为微分算子,

$$
\boldsymbol{L}(\cdot)=\left[\begin{array}{c}
-\frac{\partial^{2}}{\partial x_{1}^{2}} \\
-\frac{\partial^{2}}{\partial x_{2}^{2}} \\
-2 \frac{\partial^{2}}{\partial x_{1} \partial x_{2}}
\end{array}\right](\cdot),
$$

$M_{i i}(i=1,2)$ 是 $x_{i}$ 方向上的弯矩, $M_{12}$ 是扭矩; $\boldsymbol{D}$ 是与材 料属性、平板厚度和平板均匀性相关的常数矩阵, 对 于各向同性平板

$\boldsymbol{D}=D_{0}\left[\begin{array}{ccc}1 & v & 0 \\ v & 1 & 0 \\ 0 & 0 & \frac{1-v}{2}\end{array}\right]$,

无网格方法中, 通常采用Lagrange乘子法或罚函数法 施加本质边界条件. 采用罚函数法施加本质边界条件 时不会增加新的未知量, 因而不会增加最后形成的方
程组的阶数, 也不会破坏刚度矩阵的对称正定性, 易 于求解. 本文采用罚函数法施加本质边界条件, 其相 应的Galerkin弱形式为

$$
\begin{aligned}
& \int_{\Omega} \delta \boldsymbol{\varepsilon}_{p}^{\mathrm{T}} \cdot \sigma_{p} \mathrm{~d} \Omega-\int_{\Omega} \delta w \cdot q \mathrm{~d} \Omega-\int_{\Gamma_{t_{1}}} \delta\left(\frac{\partial w}{\partial \boldsymbol{n}}\right) \bar{M}_{n} \mathrm{~d} \Gamma \\
& -\int_{\Gamma_{t_{2}}} \delta w \overline{V_{n}} \mathrm{~d} \Gamma+\int_{\Gamma_{u_{1}}} \delta(w-\bar{w}) \cdot \alpha_{1} \cdot(w-\bar{w}) \mathrm{d} \Gamma \\
& +\int_{\Gamma_{u_{2}}} \delta\left(\frac{\partial w}{\partial \boldsymbol{n}}-\bar{\theta}\right)^{\mathrm{T}} \cdot \alpha_{2} \cdot\left(\frac{\partial w}{\partial \boldsymbol{n}}-\bar{\theta}\right) \mathrm{d} \Gamma=0,
\end{aligned}
$$

其中 $\alpha_{1}$ 和 $\alpha_{2}$ 是用来施加本质边界条件的罚因子, 是一 极大正常数, 通常取

$\alpha_{1}=\alpha_{2}=\left(1.0 \times 10^{0} \sim 1.0 \times 10^{2}\right) E$

时能够获得较高精度的解.

将式(43)的应力应变关系和式(42)的应变位移关 系代入式(46), 得

$\int_{\Omega} \delta(L w)^{\mathrm{T}} \cdot \boldsymbol{D} \cdot(L w) \mathrm{d} \Omega-\int_{\Omega} \delta w \cdot q \mathrm{~d} \Omega$

$-\int_{\Gamma_{t_{1}}} \delta\left(\frac{\partial w}{\partial \boldsymbol{n}}\right) \cdot \bar{M}_{n} \mathrm{~d} \Gamma-\int_{\Gamma_{t_{2}}} \delta w \cdot \bar{V}_{n} \mathrm{~d} \Gamma$

$+\int_{\Gamma_{u_{1}}} \delta(w-\bar{w}) \cdot \alpha_{1} \cdot(w-\bar{w}) \mathrm{d} \Gamma$

$+\int_{\Gamma_{u_{2}}} \delta\left(\frac{\partial w}{\partial \boldsymbol{n}}-\bar{\theta}\right)^{\mathrm{T}} \cdot \alpha_{2} \cdot\left(\frac{\partial w}{\partial \boldsymbol{n}}-\bar{\theta}\right) \mathrm{d} \Gamma=0$.

由式(39)可得

$$
\begin{aligned}
\boldsymbol{L} w & =\boldsymbol{L}\left(\sum_{I=1}^{N} \widetilde{\Phi}_{I} w_{I}\right)=\sum_{I=1}^{N} \boldsymbol{L}\left(\widetilde{\Phi}_{I} w_{I}\right) \\
& =\sum_{I=1}^{N}\left[\begin{array}{c}
-\frac{\partial^{2}}{\partial x_{1}^{2}} \\
-\frac{\partial^{2}}{\partial x_{2}^{2}} \\
-2 \frac{\partial^{2}}{\partial x_{1} \partial x_{2}}
\end{array}\right]\left(\operatorname{Re}\left[\Phi_{I}(z)\right] \cdot w_{I}\right) \\
& =\sum_{I=1}^{N}\left[\begin{array}{c}
-\operatorname{Re}\left[\Phi_{I, 11}\right] \\
-\operatorname{Re}\left[\Phi_{I, 22}\right] \\
-2 \operatorname{Re}\left[\Phi_{I, 12}\right]
\end{array}\right] \cdot w_{I}=\sum_{I=1}^{N} \boldsymbol{B}_{I} w_{I}=\boldsymbol{B} \cdot \boldsymbol{w},
\end{aligned}
$$

其中 $\Phi_{I, i j}(i, j=1,2)$ 是改进的复变量移动最小二乘法 形函数的二阶偏导数, 
$\boldsymbol{B}(z)=\left(\boldsymbol{B}_{1}(z), \boldsymbol{B}_{2}(z), \cdots, \boldsymbol{B}_{N}(z)\right)$,

$\boldsymbol{B}_{I}=\left[\begin{array}{c}-\operatorname{Re}\left[\Phi_{I, 11}\right] \\ -\operatorname{Re}\left[\Phi_{I, 22}\right] \\ -2 \operatorname{Re}\left[\Phi_{I, 12}\right]\end{array}\right]$.

将式(39)和(49)代入式(48), 得

$$
\begin{aligned}
\delta \boldsymbol{w}^{\mathrm{T}} \cdot\left(\int_{\Omega} \boldsymbol{B}^{\mathrm{T}} \boldsymbol{D} \boldsymbol{B} \mathrm{d} \Omega\right) \cdot \boldsymbol{w}-\delta \boldsymbol{w}^{\mathrm{T}} \cdot\left(\int_{\Omega} \widetilde{\boldsymbol{\Phi}}^{\mathrm{T}} q \mathrm{~d} \Omega\right) \\
-\delta \boldsymbol{w}^{\mathrm{T}} \cdot\left(\int_{\Gamma_{t_{1}}}\left(\frac{\partial \widetilde{\boldsymbol{\Phi}}}{\partial \boldsymbol{n}}\right)^{\mathrm{T}} \bar{M}_{n} \mathrm{~d} \Gamma\right)-\delta \boldsymbol{w}^{\mathrm{T}} \cdot\left(\int_{\Gamma_{t_{2}}} \widetilde{\boldsymbol{\Phi}}^{\mathrm{T}} \cdot \overline{V_{n}} \mathrm{~d} \Gamma\right) \\
+\delta \boldsymbol{w}^{\mathrm{T}} \cdot\left(\int_{\Gamma_{u_{1}}} \widetilde{\boldsymbol{\Phi}}^{\mathrm{T}} \cdot \alpha_{1} \cdot \widetilde{\boldsymbol{\Phi}} \mathrm{d} \Gamma\right) \cdot \boldsymbol{w} \\
-\delta \boldsymbol{w}^{\mathrm{T}} \cdot\left(\int_{\Gamma_{u_{1}}} \widetilde{\boldsymbol{\Phi}}^{\mathrm{T}} \cdot \alpha_{1} \cdot \overline{\boldsymbol{w}} \mathrm{d} \Gamma\right) \\
+\delta \boldsymbol{w}^{\mathrm{T}} \cdot\left(\int_{\Gamma_{u_{2}}}\left(\frac{\partial \widetilde{\boldsymbol{\Phi}}}{\partial \boldsymbol{n}}\right)^{\mathrm{T}} \cdot \alpha_{2} \cdot\left(\frac{\partial \widetilde{\boldsymbol{\Phi}}}{\partial \boldsymbol{n}}\right) \mathrm{d} \Gamma\right) \cdot \boldsymbol{w} \\
-\delta \boldsymbol{w}^{T} \int_{\Gamma_{u_{2}}}\left(\frac{\partial \widetilde{\boldsymbol{\Phi}}}{\partial \boldsymbol{n}}\right)^{\mathrm{T}} \cdot \alpha_{2} \cdot \bar{\theta} \mathrm{d} \Gamma=0 .
\end{aligned}
$$

由于 $\delta \boldsymbol{w}^{\mathrm{T}}$ 是任意的, 由式(52)可得最终的离散方程 为

$\overline{\boldsymbol{K}} \boldsymbol{w}=\overline{\boldsymbol{F}}$,

其中

$\overline{\boldsymbol{K}}=\boldsymbol{K}+\boldsymbol{K}^{\alpha}$,

$\overline{\boldsymbol{F}}=\boldsymbol{F}+\boldsymbol{F}^{\alpha}$,

$\boldsymbol{K}$ 是整体刚度矩阵,

$$
\boldsymbol{K}=\int_{\Omega} \boldsymbol{B}^{\mathrm{T}} \cdot \boldsymbol{D} \cdot \boldsymbol{B} \mathrm{d} \Omega
$$

$\boldsymbol{K}^{\alpha}$ 是整体罚函数矩阵,

$$
\begin{aligned}
\boldsymbol{K}^{\alpha}= & \int_{\Gamma_{u_{1}}} \widetilde{\boldsymbol{\Phi}}^{\mathrm{T}} \cdot \alpha_{1} \cdot \widetilde{\boldsymbol{\Phi}} \mathrm{d} \Gamma \\
& +\int_{\Gamma_{u_{2}}}\left(\frac{\partial \widetilde{\boldsymbol{\Phi}}}{\partial \boldsymbol{n}}\right)^{\mathrm{T}} \cdot \alpha_{2} \cdot\left(\frac{\partial \widetilde{\boldsymbol{\Phi}}}{\partial \boldsymbol{n}}\right) \mathrm{d} \Gamma ;
\end{aligned}
$$

$\boldsymbol{F}$ 是整体外力向量,

$\boldsymbol{F}=\int_{\Omega} \widetilde{\boldsymbol{\Phi}}^{\mathrm{T}} q \mathrm{~d} \Omega+\int_{\Gamma_{t_{1}}}\left(\frac{\partial \widetilde{\boldsymbol{\Phi}}}{\partial \boldsymbol{n}}\right)^{\mathrm{T}} \bar{M}_{n} \mathrm{~d} \Gamma+\int_{\Gamma_{t_{2}}} \widetilde{\boldsymbol{\Phi}}^{\mathrm{T}} \bar{V}_{n} \mathrm{~d} \Gamma$
$F^{\alpha}$ 是由本质边界条件产生的向量,

$$
\boldsymbol{F}^{\alpha}=\int_{\Gamma_{u_{1}}} \widetilde{\boldsymbol{\Phi}}^{\mathrm{T}} \cdot \alpha_{1} \cdot \bar{w} \mathrm{~d} \Gamma+\int_{\Gamma_{u_{2}}}\left(\frac{\partial \widetilde{\boldsymbol{\Phi}}}{\partial \boldsymbol{n}}\right)^{\mathrm{T}} \cdot \alpha_{2} \cdot \bar{\theta} \mathrm{d} \Gamma
$$

\section{5 数值算例}

本文建立的Kirchhoff板弯曲问题的改进的复变量 无单元Galerkin方法, 其数值计算流程如下.

(1) 输入已知参数, 包括问题域的几何属性和材料 常数.

(2) 对于给定的Kirchhoff板弯曲问题, 确定坐标系, 在求解域 $\Omega$ 及其边界 $\Gamma$ 上布置 $n$ 个节点 $z_{I}, I=1,2, \cdots, n$, 并对其进行编号.

(3) 形成用于数值积分的背景积分网格.

(4) 建立边界 $\Gamma$ 上的积分网格信息.

(5) 根据背景网格和边界, 生成Gauss积分点并计 算相应Gauss积分点信息(包括Gauss积分点的坐标、 积分权重和相应的Jacobi行列式 $J \mid)$.

(6) 形成矩阵 $\boldsymbol{K}$ 和载荷列向量 $\boldsymbol{F}$ 第一项 $\boldsymbol{F}^{(1)}$ :

1) 对每一个背景积分网格进行循环:

a) 对背景网格内的Gauss积分点进行循环;

b) 若Gauss积分点在 $\Omega$ 内, 则进行步骤(c) 一步骤(f), 否则直接进行步骤(f);

c) 基于一定的规则, 确定影响域覆盖当前Gauss积 分点 $z_{Q}$ 的节点;

d) 分别计算影响域覆盖Gauss积分点 $z_{Q}$ 的所有节 点 $Z_{I}$ 处的形函数 $\Phi_{I}\left(z_{Q}\right)$ 及其二阶导数 $\Phi_{I, i j}\left(z_{Q}\right)$ 的值;

e) 根据式(56)和(58)第一项计算当前Gauss积分点 $z_{Q}$ 对矩阵 $\boldsymbol{K}$ 和 $\boldsymbol{F}^{(1)}$ 元素的贡献;

f) 结束Gauss积分点的循环.

2) 结束背景积分网格的循环.

(7) 边界 $\Gamma_{t_{1}}$ 上积分: 与步骤(6)类似, 根据式(58)第 二项计算载荷列向量的第二项, 记为 $\boldsymbol{F}^{(2)}$.

(8) 边界 $\Gamma_{t_{2}}$ 上积分: 与步骤(6)类似, 根据式(58)第 三项计算出载荷列向量的第三项, 记为 $\boldsymbol{F}^{(3)}$.

(9) 边界 $\Gamma_{u_{1}}$ 上积分: 与步骤 (6) 类似, 分别根 据式(57)第一项和式(59)第一项计算, 分别记为矩 阵 $\boldsymbol{K}^{\alpha_{1}}$ 和列向量 $\boldsymbol{F}^{\alpha_{1}}$.

(10) 边界 $\Gamma_{u_{2}}$ 上积分：与步骤 (6) 类似, 分别根 据式(57)第二项和式(59)第二项计算, 分别记为矩 
阵 $\boldsymbol{K}^{\alpha_{2}}$ 和列向量 $\boldsymbol{F}^{\alpha_{2}}$.

(11) 将步骤(6)计算所得的 $\boldsymbol{K}$ 矩阵与步骤(9), (10) 计算所得的 $\boldsymbol{K}^{\alpha_{1}}, \boldsymbol{K}^{\alpha_{2}}$ 矩阵相加, 得到最后的求解刚度 阵 $\overline{\boldsymbol{K}}$.

(12) 将步骤(6)-(10)计算所得的列向量 $\boldsymbol{F}^{(1)}, \boldsymbol{F}^{(2)}$,

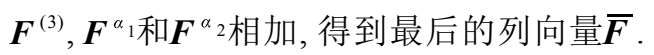

(13) 求解代数数方程组(53), 得到 $n$ 个节点挠度函 数的名义数值解 $\widehat{\boldsymbol{w}}$.

(14) 由 $\widehat{w}$ 拟合出 $\Omega$ 内 $n$ 个节点的挠度函数数值解.

(15) 输出节点挠度.

以下利用本文建立的Kirchhoff板问题的改进的复 变量无单元Galerkin方法对 4 个不同边界条件的Kirchhoff板进行求解, 说明本文方法的优点.

数值算例中, 杨氏模量为 $E=2.1 \times 10^{11} \mathrm{~N} / \mathrm{m}^{2}$, 泊 松比为 $v=0.3$, 板的厚度为 $h=0.01 \mathrm{~m}$. 背景积分网格 的数值积分采用 $4 \times 4$ 点Gauss 积分.

\section{1 四边形简支方板}

采用本文建立的Kirchhoff板问题的改进的复变量 无单元Galerkin方法对本算例进行数值分析以确定本 文方法如何选取适当的基函数、权函数、影响域比 例参数、节点分布和罚因子. 然后, 通过分析节点的 挠度和应变能, 分析本文方法的收玫性.

挠度范数 $\|w\|$ 和应变能范数 $\|e\|$ 分别定义为

$\|w\|=\left(\int_{\Omega} w^{2} \mathrm{~d} \Omega\right)^{\frac{1}{2}}$,

$\|e\|=\left[\frac{D_{0}}{2} \int_{\Omega}\left(\nabla^{2} w\right)^{2} \mathrm{~d} \Omega\right]^{\frac{1}{2}}$.

挠度\| $\mid w \|$ 和应变能 $\|e\|$ 相对误差分别定义为

$r_{w}=\frac{\left\|w^{n}-w^{e}\right\|}{\left\|w^{e}\right\|}$

$r_{e}=\frac{\left\|e^{n}-e^{e}\right\|}{\left\|e^{e}\right\|}$

其中 $w^{n}$ 是改进的复变量无单元Galerkin方法的挠度数 值解, $e^{n}$ 是由改进的复变量无单元Galerkin方法得到的 应变能数值解, $w^{e}$ 和 $e^{e}$ 分别表示相应板弯曲问题的解 析解.

考虑受均布载荷作用的四边简支方形Kirchhoff板
(见图2), 其解析解为 ${ }^{[32]}$

$w=\frac{16 q_{0}}{\pi^{6} D_{0}} \sum_{m=1,3, \cdots}^{\infty} \sum_{n=1,3, \cdots}^{\infty} \frac{\sin \frac{m \pi x_{1}}{a} \cdot \sin \frac{n \pi x_{2}}{a}}{m n\left(\frac{m^{2}}{a^{2}}+\frac{n^{2}}{a^{2}}\right)}$,

其中 $q_{0}$ 是均布载荷集度, $a$ 是方板边长. 在本算例中, $q_{0}=1000 \mathrm{~N} / \mathrm{m}^{2}, a=1.0$.

图3和4分别给出了无单元Galerkin方法和改进的

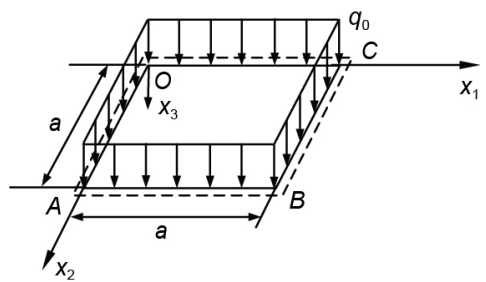

图 2 受均布载荷作用的四边简支方板

Figure 2 A simply supported square plate subjected to a uniformly distributed load.

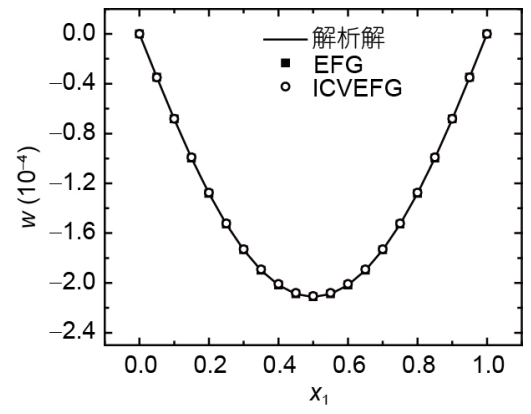

图 $3 x_{2}=0.5$ 处中面挠度的数值解

Figure 3 The numerical solutions of the deflection on the middle plane when $x_{2}=0.5$.

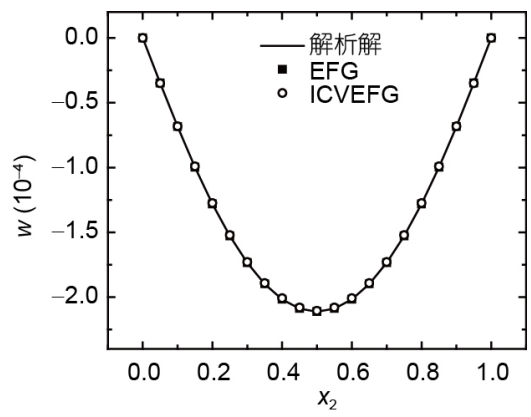

图 $4 x_{1}=0.5$ 处中面挠度的数值解

Figure 4 The numerical solutions of the deflection on the middle plane when $x_{1}=0.5$. 
复变量无单元Galerkin方法计算得到的Kirchhoff板中 面 $x_{2}=0.5$ 和 $x_{1}=0.5$ 处挠度的数值解. 可以看出, 本文 改进的复变量无单元Galerkin方法的数值解与无单元 Galerkin方法的数值解和解析解相吻合.

由 Kirchhoff 板弯曲问题的 Galerkin 弱形式 (见式(48)) 可以看出, 求解域内积分中形函数含挠 度 $w$ 的二阶导数, 而在求解域边界积分中形函数含挠 度的一阶导数, 为保证在求解域及其边界上积分存 在, 挠度试函数必须选择 $C^{1}$ 连续可导函数. 板挠度 $w$ 的 改进的复变量移动最小二乘法逼近函数的连续可导 性是由基函数和权函数的连续可导性确定, 所以基 函数必须具有二阶可导的性质, 在这里分别选择二 次、三次和四次多项式为基函数及三次、四次和五 次样条权函数.

采用 $7 \times 7,10 \times 10$ 和 $21 \times 21$ 这 3 种规则节点分布来 计算改进的复变量无单元Galerkin方法的相对误差. 这里图5-8中的尺度 $h$ 表示为相邻两节点之间的距离. 图5和6分别给出了挠度 $\|w\|$ 和应变能 \|\| 的相对误差. 可 以看出通过改进的复变量无单元Galerkin方法得到的 挠度和应变能具有较高的收玫性. 另外, 相对于二次 多项式, 基函数取三次多项式和四次多项式能获得更 高的精度,但是计算所需时间更多.

由改进的复变量移动最小二乘法的逼近函数可 知, 为获得较高的计算精度, 需选取合适的权函数. 图7和8给出了分别采用三次、四次和五次样条权函 数计算得到的挠度和应变能的相对误差. 可以看出, 采用三次和四次样条权函数计算得到的数值解比五 次样条权函数的数值解计算精度更高.

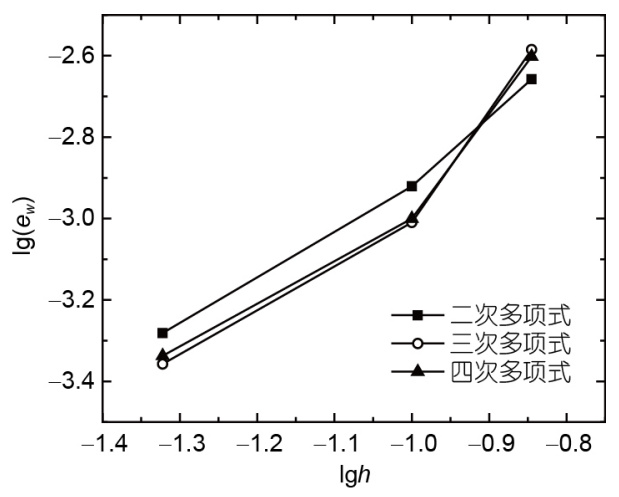

图 5 不同基函数时挠度数值解的相对误差

Figure 5 The relative errors of the numerical solutions of the deflections with different basis functions.

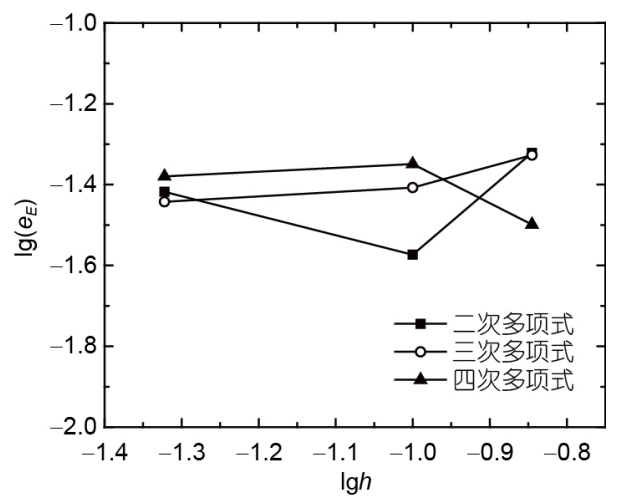

图 6 不同基函数时应变能数值解的相对误差

Figure 6 The relative errors of the numerical solutions of the strain energy with different basis functions.

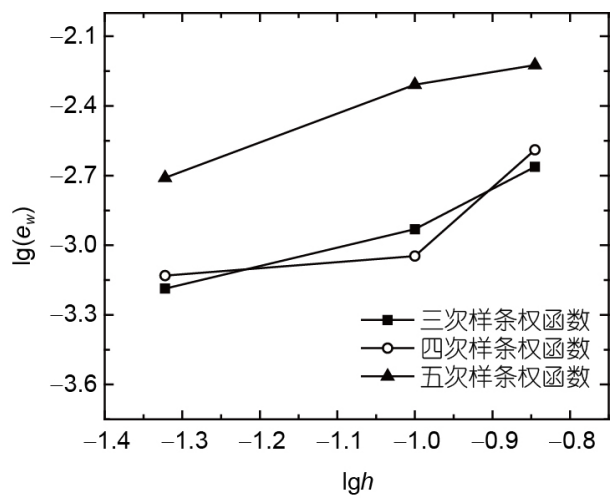

图 7 不同权函数时挠度数值解的相对误差

Figure 7 The relative errors of the numerical solutions of the deflections with different weight functions.

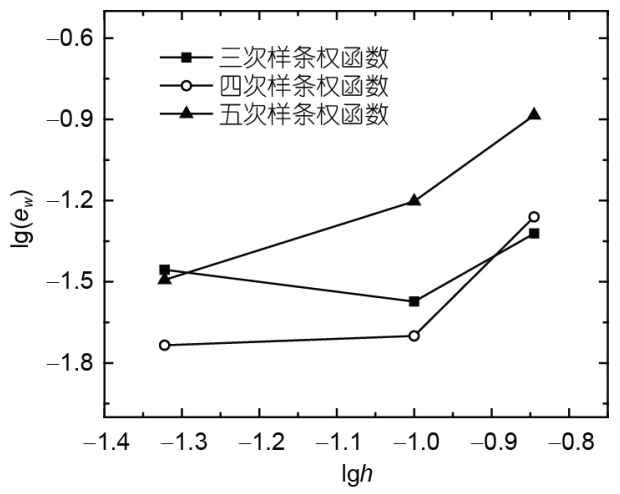

图 8 不同权函数时应变能数值解的相对误差

Figure 8 The relative errors of the numerical solutions of the strain energy with different weight functions.

由图5-8可以看出, 采用本文改进的复变量无单 元Galerkin方法进行计算时, 不论是未知函数还是其 
导数均可得到较高精度的数值解. 另外, 由于逼近函 数的连续性与权函数的连续性相关, 如果权函数的连 续性阶次高于基函数的连续性阶次, 那么逼近函数的 连续性阶次将和权函数的连续性阶次相同。

综合理论分析以及图 5-8 的数值结果, 本文以下 算例将采用二次多项式基函数和三次样条权函数进 行计算.

接下来讨论在不同的节点分布下影响域比例参数 $d_{\text {max }}$ 对数值结果的影响. 图9中给出挠度范数 $\|w\|$ 和 $d_{\text {max }}$ 的关系, 可以看出, 当 $d_{\text {max }}$ 取值为4.2-4.4时可以得到计 算精度较高的数值解. 在本文以下算例中, 取 $d_{\text {max }}=4.4$.

本节中还讨论了罚因子 $\alpha$ 对改进的复变量无单 元 Galerkin方法数值解的影响. 如图 10 所示, 在 $7 \times 7$, $9 \times 9,11 \times 11,13 \times 13,15 \times 15,17 \times 17$ 和 $19 \times 19$ 节点分布 下, 不同罚因子的挠度相对误差\|w\|结果. 可以看出 当 $\alpha=(0.1 \sim 1) \times E$ 时, Kirchhoff板的数值解有较好的 结果. 因此, 在后面的例子中, 取 $\alpha=0.1 \times E$.

最后, 图11给出了不同节点分布时, 采用改进的 复变量无单元Galerkin方法计算得到的挠度数值解的 相对误差. 可以看出, 随着节点数的增加, 计算精度也 会随之提高. 因而, 本文建立的Kirchhoff板弯曲问题 的改进复变量无单元Galerkin方法具有较好的收敛性.

\section{2 受均布弯矩的四边简支矩形Kirchhoff板}

考虑对边受均布弯曲力偶 $M_{0}$ 作用的四边简支的 矩形Kirchhoff板, 如图12所示.

对边均布弯矩作用的四边简支板, $x_{2}=0$ 处挠度 的解析解为

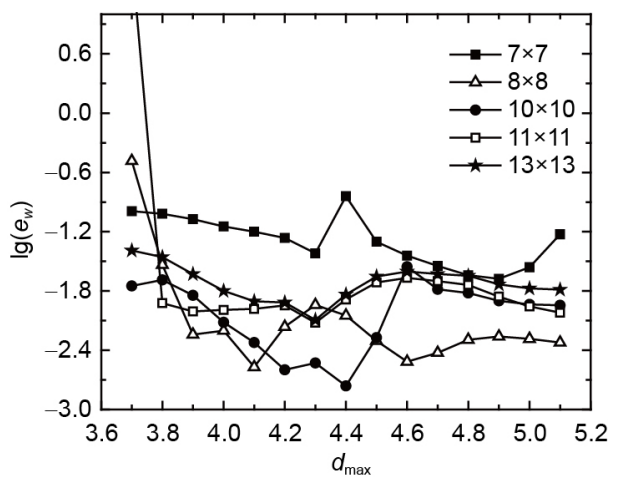

图 9 影响域比例参数不同时挠度数值解的相对误差

Figure 9 The relative errors of the numerical solutions of the deflections with different scaling factors of the influence domain.

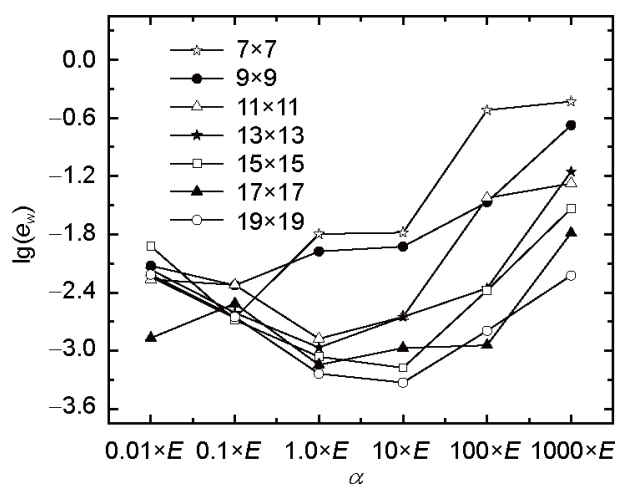

图 10 不同罚因子时挠度数值解的相对误差

Figure 10 The relative errors of the numerical solutions of the deflections with different penalty factors.

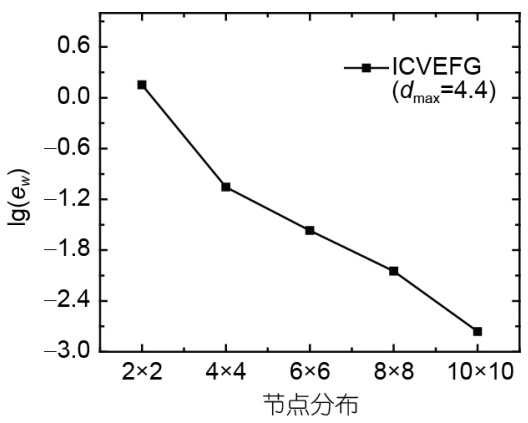

图 11 挠度数值解的收敛性

Figure 11 The convergence of the numerical solutions of the deflections.

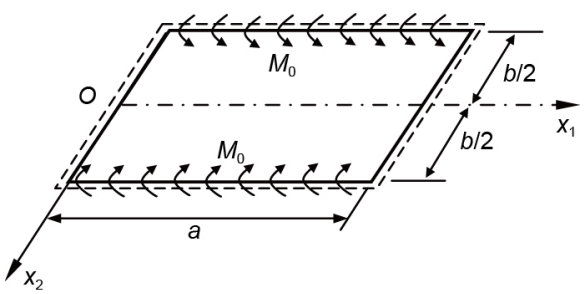

图 12 四边简支的矩形Kirchhoff板

Figure 12 A rectangular Kirchhoff plate simply supported on four edges.

$(w)_{x_{2}=0}=\frac{2 M_{0} a^{2}}{\pi^{3} D_{0}} \sum_{m=1}^{\infty} \frac{1}{m^{3}} \frac{\alpha_{m} \text { th } \alpha_{m}}{\operatorname{ch} \alpha_{m}} \sin \frac{m \pi x_{1}}{a}$,

其中

$\alpha_{m}=\frac{m \pi b}{2 a}, m=1,3,5, \cdots$,

在本算例中, 矩形板长 $a=2$, 板宽 $b=1$, 载荷集度 
为 $M_{0}=1000 \mathrm{Nm} / \mathrm{m}$. 基于 5.1 算例的结论, 取影响域 比例参数 $d_{\max }=4.4$, 权函数为三次样条函数, 罚因子 取 $\alpha_{1}=2.1 \times 10^{10}$. 为了验证本文方法的有效性, 图13给 出了改进的复变量无单元Galerkin方法得到的沿 $x_{1}$ 方 向的中面挠度的数值解和解析解的比较. 可以看出本 文改进的复变量无单元Galerkin方法的数值解与解析 解吻合.

\section{3 受线性分布载荷作用的简支方板}

考虑线性分布载荷下的四边简支方板(见图 14), 其挠度的解析解为

$w=\frac{8 q_{0} a^{4}}{D_{0} \pi^{6}} \sum_{m=1 n=1}^{\infty} \sum_{n=1}^{\infty} \frac{(-1)^{m+1}}{m n\left(m^{2}+n^{2}\right)^{2}} \sin \frac{m \pi x_{1}}{a} \sin \frac{n \pi x_{2}}{a}$,

$n=1,3,5, \cdots$,

其中 $q_{0}$ 是线性分布载荷集度的最大值.

本例取线性分布载荷的最大载荷集度为 $q_{0}=1000 \mathrm{~N} / \mathrm{m}^{2}$, 影响域比例参数为 $d_{\max }=4.4$, 罚因子 为 $\alpha_{1}=2.1 \times 10^{10}$.

图15和16分别给出了 $\left(x_{1}, 0.5\right)$ 和 $\left(0.5, x_{2}\right)$ 处本文

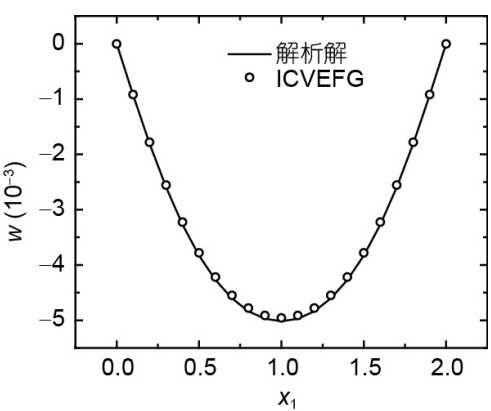

图 $13 x_{2}=0$ 处的中面挠度的数值解

Figure 13 The numerical solutions of the deflection on the middle plane when $x_{2}=0$.

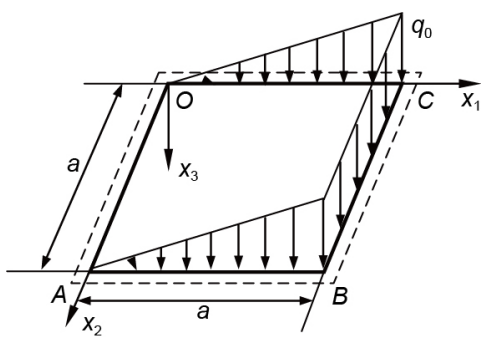

图 14 线性分布载荷作用下的四边简支方板

Figure 14 A square plate simply supported on four edges under linearly distributed load.
改进的复变量无单元Galerkin方法得到的中面挠度的 数值解. 同样可以看出, 本文建立的改进的复变量无 单元Galerkin方法的数值解与解析解吻合.

\section{4 四边固支的矩形Kirchhoff板}

考虑均布载荷作用下的四边固支矩形Kirchhoff板 (见图17). 矩形板长 $a=2$ ，板宽 $b=1$; 均布压力集度

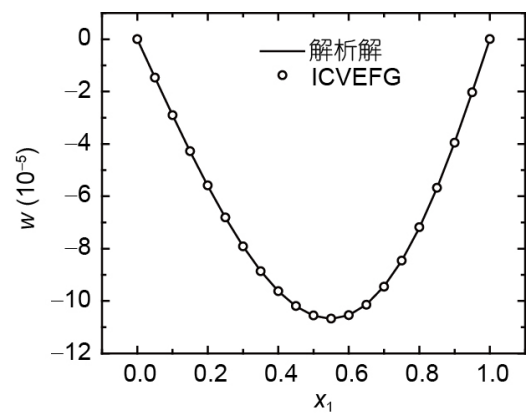

图 15 点 $\left(x_{1}, 0.5\right)$ 处中面挠度的数值解

Figure 15 The numerical solutions of the deflection on the middle plane at points $\left(x_{1}, 0.5\right)$.

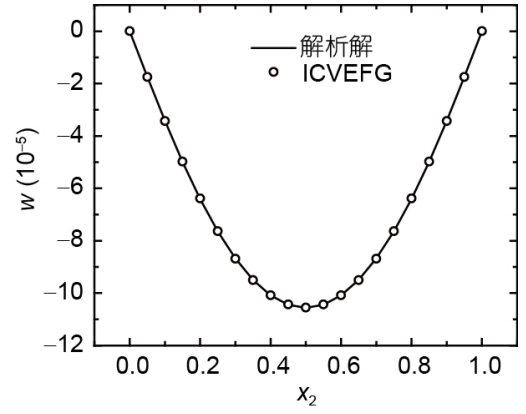

图 16 点 $\left(0.5, x_{2}\right)$ 处中面挠度的数值解

Figure 16 The numerical solutions of the deflection on the middle plane at points $\left(0.5, x_{2}\right)$.

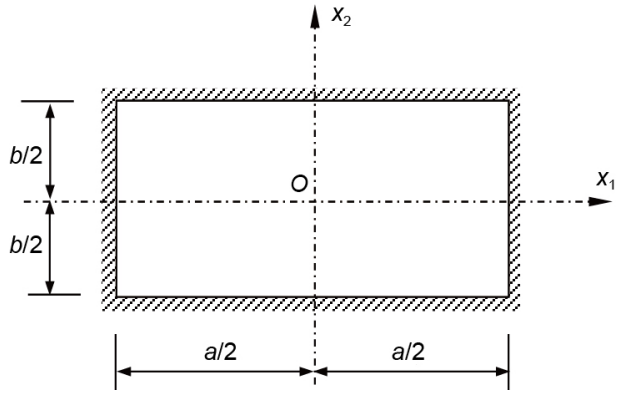

图 17 四边固支的矩形Kirchhoff板

Figure 17 A rectangular Kirchhoff plate with four clamped edges. 
$q_{0}=1000 \mathrm{~N} / \mathrm{m}^{2}$.

对于均布载荷作用的四边固支矩形Kirchhoff板, 其挠度的解析解为

$w=\frac{a^{4} b^{4} q_{0}}{\pi^{4}\left[3\left(a^{4}+b^{4}\right)+2 a^{2} b^{2}\right] D_{0}} \cos ^{2} \frac{\pi x_{1}}{a} \cos ^{2} \frac{\pi x_{2}}{b}$.

本算例采用改进的复变量移动最小二乘法计算 Kirchhoff板弯曲问题时, 在矩形板求解域均匀布置了 $21 \times 11$ 个节点, 基函数采用二次多项式, 权函数为三次 样条函数, 权函数影响域比例参数为 $d_{\max }=4.1$, 罚因子 为 $\alpha_{1}=3.1 \times 10^{15}, \alpha_{2}=2.1 \times 10^{12}$. 图 18 和 19 分别给出 了 $x_{1}=0$ 和 $x_{2}=0$ 处中面挠度的数值解.

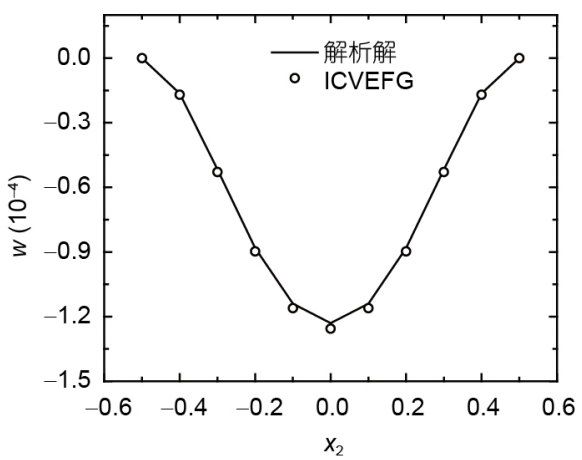

图 $18 x_{1}=0$ 处中面挠度的数值解

Figure 18 The numerical solutions of the deflection on the middle plane when $x_{1}=0$.

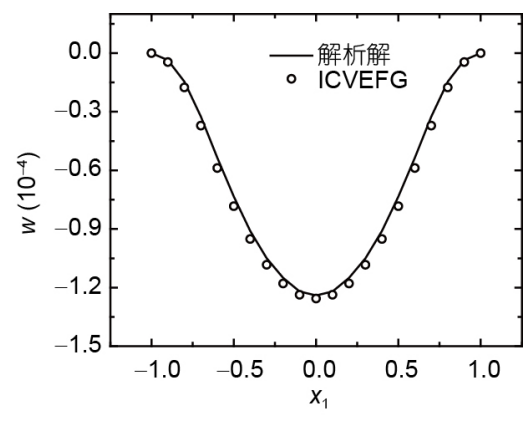

图 $19 x_{2}=0$ 处中面挠度的数值解

Figure 19 The numerical solutions of the deflection on the middle plane when $x_{2}=0$.

\section{6 结论}

本文建立了Kirchhoff板弯曲问题的改进的复变量 无单元Galerkin方法, 并讨论了本文方法如何选取合 适的基函数、权函数、影响域比例参数、节点分布 和罚因子以保证数值解的精度. 算例表明, 本文方法 具有较好的收敛性和较高的计算精度.

虽然本文使用各向同性和均匀板厚简化了问题, 但是本文的Kirchhoff板弯曲问题的改进的复变量无单 元Galerkin方法可以推广到任意材料属性的板弯曲问 题. 此外, 本文的计算公式可灵活适应任意计算区域 任意节点分布, 因此能够方便地通过加密节点提高计 算精度, 有利于发展相关的自适应算法并应用于工程 实际问题.

\section{参考文献}

1 Hein P. Diffuse Element Method Applied to Kirchhoff Plates. Technical Report, Department of Civil Engineering, Northwestern University, Evanston. 1993

2 Krysl P, Belytschko T. Analysis of thin plates by the element-free Galerkin method. Comput Mech, 1995, 17: 26-35

3 Li S, Hao W, Liu W K. Numerical simulations of large deformation of thin shell structures using meshfree methods. Comput Mech, 2000, 25: $102-116$

4 Liu G R, Chen X L. A mesh-free method for static and free vibration analyses of thin plates of complicated shape. J Sound Vib, 2001, 241: $839-855$

5 Gu Y T, Liu G R, A meshless local Petrov-Galerkin (MLPG) formulation for static and free vibration analyses of thin plates. Comp Model Eng Sci, 2001, 2: 463-476

6 Sladek J, Sladek V. A meshless method for large deflection of plates. Comput Mech, 2003, 30: 155-163

7 Sladek J, Sladek V, Zhang C, et al. Analysis of orthotropic thick plates by meshless local Petrov-Galerkin (MLPG) method. Int J Numer Meth Engng, 2006, 67: 1830-1850

8 Chen L, Cheng Y M. The complex variable reproducing kernel particle method for elasto-plasticity problems. Sci China-Phys Mech Astron, 2010, 53: 954-965

9 Chen L, Cheng Y M, Ma H P. The complex variable reproducing kernel particle method for the analysis of Kirchhoff plates. Comput Mech, 2015, 55: 591-602 
10 Cheng Y M, Chen M J, A boundary element-free method for linear elasticity (in Chinese). Acta Mech Sin, 2000, 25: 181-186 [程玉民, 陈美娟. 弹性力学的一种边界无单元法. 力学学报, 2000, 25: 181-186]

11 Wang J F, Sun F X, Cheng Y M, et al. Error estimates for the interpolating moving least-squares method. Appl Math Computation, 2014, 245: $321-342$

12 Sun F X, Wang J F, Cheng Y M, et al. Error estimates for the interpolating moving least-squares method in $n$-dimensional space. Appl Numer Math, 2015, 98: 79-105

13 Cheng Y, Li J. A complex variable meshless method for fracture problems. Sci China Ser G-Phys Mech Astron, 2006, 49: 46-59

14 Cheng Y M, Peng M J, Li J H. The complex variable moving least-square approximation and its application (in Chinese). Chin J Theor Appl Mech, 2005, 37: 719-723 [程玉民, 彭妙娟, 李九红. 复变量移动最小二乘法及其应用. 力学学报, 2005, 37: 719-723]

15 Li D M, Peng M J, Cheng Y M. The complex variable element-free Galerkin (CVEFG) method for elastic large deformation problems (in Chinese). Sci Sin-Phys Mech Astron, 2011, 41: 1003-1014 [李冬明, 彭妙娟, 程玉民. 弹性大变形问题的复变量无单元Galerkin方法. 中国科学: 物 理学力学 天文学, 2011, 41: 1003-1014]

16 Ren H, Cheng J, Huang A. The complex variable interpolating moving least-squares method. Appl Math Comput, 2012, 219: 1724-1736

17 Zhang Z, Wang J F, Cheng Y M, et al. The improved element-free Galerkin method for three-dimensional transient heat conduction problems. Sci China-Phys Mech Astron, 2013, 56: 1568-1580

18 Zhang Z, Li D M, Cheng Y M, et al. The improved element-free Galerkin method for three-dimensional wave equation. Acta Mech Sin, 2012, 28: $808-818$

19 Zhang Z, Hao S Y, Liew K M, et al. The improved element-free Galerkin method for two-dimensional elastodynamics problems. Eng Anal Bound Elem, 2013, 37: 1576-1584

20 Peng M J, Li R X, Cheng Y M. Analyzing three-dimensional viscoelasticity problems via the improved element-free Galerkin (IEFG) method. Eng Anal Bound Elem, 2014, 40: 104-113

21 Cheng Y, Bai F, Liu C, et al. Analyzing nonlinear large deformation with an improved element-free Galerkin method via the interpolating moving least-squares method. Int J Comp Mat Sci Eng, 2016, 05: 1650023

22 Sun F, Wang J, Cheng Y. An improved interpolating element-free Galerkin method for elastoplasticity via nonsingular weight functions. Int J Appl Mech, 2016, 08: 1650096

23 Peng M, Liu P, Cheng Y. The complex variable element-free Galerkin (CVEFG) method for two-dimensional elasticity problems. Int J Appl Mech, 2009, 01: 367-385

24 Peng M, Li D, Cheng Y. The complex variable element-free Galerkin (CVEFG) method for elasto-plasticity problems. Eng Struct, 2011, 33: $127-135$

25 Cheng Y, Wang J, Li R. The complex variable element-free Galerkin (CVEFG) method for two-dimensional elastodynamics problems. Int J Appl Mech, 2012, 04: 1250042

26 Cheng Y M, Li R X, Peng M J. Complex variable element-free Galerkin method for viscoelasticity problems. Chin Phys B, 2012, 21: 090205

27 Weng Y J, Zhang Z, Cheng Y M. The complex variable reproducing kernel particle method for two-dimensional inverse heat conduction problems. Eng Anal Bound Elem, 2014, 44: 36-44

28 Li D M, Liew K M, Cheng Y M. Analyzing elastoplastic large deformation problems with the complex variable element-free Galerkin method. Comput Mech, 2014, 53: 1149-1162

29 Deng Y, Liu C, Peng M, et al. The interpolating complex variable element-free Galerkin method for temperature field problems. Int J Appl Mech, 2015, 07: 1550017

30 Cheng Y M, Bai F N, Peng M J. A novel interpolating element-free Galerkin (IEFG) method for two-dimensional elastoplasticity. Appl Math Model, 2014, 38: 5187-5197

31 Ren H, Cheng Y. A new element-free Galerkin method based on improved complex variable moving least-squares approximation for elasticity. Int J Comp Mat Sci Eng, 2012, 01: 1250011

32 Timoshenko S, Woinowsky-Krieger S. Theory of Plates and Shells. 2nd ed. New York: McGraw-Hill, 1959 


\title{
The improved complex variable element-free Galerkin method for the analysis of Kirchhoff plates
}

\author{
WANG BinHua ${ }^{1}$, MA YongQi ${ }^{1,2},{\text { FENG } \mathrm{Wei}^{1} \text { \& CHENG YuMin }}^{1 *}$ \\ ${ }^{1}$ Shanghai Institute of Applied Mathematics and Mechanics, Shanghai University, Shanghai 200072, China; \\ ${ }^{2}$ Department of Mechanics, Shanghai University, Shanghai 200444, China
}

Based on the improved complex variable moving least-squares (ICVMLS) approximation, the improved complex variable element-free Galerkin (ICVEFG) method for the bending problem of Kirchhoff plate is presented. Compared with the moving least-squares (MLS) approximation, in the ICVMLS approximation, the approximation function of two-dimensional problems can be obtained with one-dimensional basis function, then the computational efficiency of the shape functions is higher. Compared with the meshless methods based on the MLS approximation, under the same node distributions, the ones using the ICVMLS approximation can obtain the solutions with higher computational accuracy; and under the similar computational accuracy, the ones using the ICVMLS approximation have higher computational efficiency. The ICVMLS approximation is used to form the approximation function of the deflection of a Kirchhoff plate, the Galerkin weak form of the bending problem of Kirchhoff plates is adopted to obtain the discretized system equations, and the penalty method is employed to enforce the essential boundary conditions, then the corresponding formulae of the ICVEFG method for the bending problem of Kirchhoff plates are presented. By computing and analyzing four typical examples, it is shown that the ICVEFG method of Kirchhoff plates in this paper is efficient. And the computational precision of the numerical solutions is analyzed to select the basis function, weight function, scaling factor, node distribution and penalty factor in the ICVEFG method. Numerical examples show that the method in this paper has better convergence and higher accuracy. When the quadratic polynomial basis function and the cubic or quartic spline weight function are used, and $d_{\max }=4.2-4.4$, the ICVEFG method of Kirchhoff plates in this paper can obtain the solutions with high computational accuracy. And more nodes are distributed in the problem domain, higher computational accuracy of the solution will obtained, which shows that the method in this paper has better convergence.

meshless method, improved complex variable moving least-squares approximation, complex variable element-free Galerkin method, improved complex variable element-free Galerkin method, Kirchhoff plate

PACS: 45.10.-b, 46.15.-x, 46.25.-y, 46.70.De

doi: $10.1360 /$ SSPMA2016-00437 